\title{
David Oliver: Conveyor belt medicine
}

\author{
David Oliver consultant in geriatrics and acute general medicine
}

Berkshire

Over the New Year period I worked three 12 hour stints on the acute medical unit. What I saw, heard, and felt over those days, and over many others this winter, was nothing unusual for colleagues in acute care throughout the NHS. What it perhaps resembled most was an out-of-control conveyor belt, of the kind used to tragicomic effect by Charlie Chaplin in the 1936 film Modern Times. ${ }^{1}$ This one wasn't carrying parts needing screws, however, but sick, frightened, or confused patients and their worried relatives. The operatives in this case weren't depression era factory workers but clinical staff, struggling to deal with the relentless procession and unable to stop it.

We know that each clinical encounter really matters to patients and their relatives. It will be remembered and discussed by them, not least if things go wrong. We know that decisions, omissions, demeanour, and the time we give people can help or harm and can enhance or worsen experiences. These are often fraught with managed or balanced risks.

We seem to have several conveyor belts to manage simultaneously as we run from one to the other in a forlorn attempt to stay on top of the work. We get phone calls for referral or advice, or emergency crash calls that distract and drag people away, mid-task. Patients we saw earlier in the day can deteriorate or require further assessment or admin. Updates remind us of negative bed capacity and our imperative to find more patients to send home sooner. Bed shortages have turned decisions to admit people overnight into a thought crime.

Data on hospital attendances and admissions tell us that none of these pressures is unique to winter, and numbers rise yearly. ${ }^{2}$ But a slowdown in community health and care services over Christmas and New Year can mean even more beds being taken out of commission by stranded patients, ${ }^{3}$ with backlogs taking weeks to clear.

Local surges of flu and respiratory viruses can put more pressure on hospitals. Outbreaks of gastrointestinal viruses lead to ward closures and further difficulties in discharging patients. Overcrowding makes these outbreaks hard to tackle. Staff go off sick with the same problems.

We do much of our work in very public areas, being overheard and watched, while trying to maintain a semblance of confidentiality but also feeling the pressure of dozens of eyes burning into us, in the hope that we'll be coming to them next. We're approached and interrupted ${ }^{4}$ by people keen for reassurance and updates: even when they're not our patients we're seen as interchangeable. Concentration is broken, and errors become more likely. If we assert ourselves we risk complaints that we've been rude or dismissive.

Our minds are often partly on the next, or sicker, patient as we try to stop the conveyor belt from swamping us. We can't give people the individual, structured time and attention we've been trained for or focus on the clinician-patient relationship.

There are compensations. When I go home I'm never in doubt that I've done something of value. There can be tremendous camaraderie, mutual support, and gallows humour among teams of clinical staff and operational managers working together, bonded by such circumstances in a common purpose. And there's real skill in being able to work at pace, accept risk, and coordinate the flow of work.

But I've seen too many staff in tears, at their human limits-not because of the inherent nature of clinical work but because of a system and workforce not designed for this volume of activity, expectation, or pressure.

When items on Chaplin's conveyor belt started to fall off and break, his line manager just drove him harder. He had a nervous breakdown and ended up being swallowed by the machine-only in his case it was fictional, and funny.

Competing interests: See www.bmj.com/about-bmj/freelance-contributors. Provenance and peer review: Commissioned; not externally peer reviewed.

1 Wikipedia. Modern Times (film). https://en.wikipedia.org/wiki/Modern_Times_(film). 2 Nuffield Trust. A\&E waiting times. 2019. https://www.nuffieldtrust.org.uk/resource/a-ewaiting-times.

3 Oliver D. David Oliver: Guidance tools won't solve long hospital stays. BMJ 2019;367:15870 10.1136/bmj.15870 31597635

4 Berg LM, Källberg A-S, Göransson KE, Östergren J, Florin J, Ehrenberg A. Interruptions in emergency department work: an observational and interview study. BMJ Qual Sa 2013;22:656-63. https://qualitysafety.bmj.com/content/22/8/656.short. 10.1136/bmjqs-2013-001967 23584208

Published by the BMJ Publishing Group Limited. For permission to use (where not already granted under a licence) please go to http://group.bmj.com/group/rights-licensing/ permissions 
